"This," I answered, "is the allocation of awards to consultants in Scotland." At his request I passed it over and he scowled at it for some minutes.

"I see," he said, "that pathologists and physicians rank almost side by side in Scotland. How does that come about?"

With what tact and care I could muster I emphasised that departments of pathology were very highly regarded in Scotland and that some who aspired to become consultants in pathology gladly enough turned to clinical medicine or surgery if they found themselves falling behind in the promotion race; and indeed that many budding physicians and surgeons regarded a year or two in pathology as a good way of preparing for their clinical careers. I also explained that the four university departments of pathology in Scotland were by no means ivory towers but accepted in full a service commitment to their teaching hospitals and to nearby peripheral hospitals as well.

"Remarkable," he said; "and I see that your new college has its share of that breed."

"I promise," he said as we parted, "that due weight will be given to your arguments." He was as good as his word. Before he died in December 1966 a visible and upward trend in favour of pathologists was well established.

From our one and only meeting I concluded that he was a much warmer man than was generally realised.

\title{
Incidence of unsuspected fractures in traumatic effusions of the elbow joint
}

\author{
D J W MOREWOOD
}

Radiological signs of an effusion of the elbow are the presence of the posterior fat pad and elevation of the anterior fat pad in a lateral radiograph. It has been suggested that when an elbow effusion is present after trauma a fracture is also likely to be present. ${ }^{1.3}$ The aim of this study was to determine the incidence of fractures not seen in the radiograph at presentation when a traumatic elbow effusion was present radiologically.

\section{Patients, methods, and results}

All patients who had anteroposterior and lateral radiographs taken of the elbow after trauma and in whom the radiographs showed effusion but no clear fracture returned for repeat anteroposterior and lateral radiography of the elbow seven to 14 days after the initial trauma. The study comprised 35 patients ( 19 male and 16 female). The age range was $9-73$ years, nine patients being under 16 .

At presentation 31 radiographs showed elevation of both fat pads and four showed elevation of only the anterior fat pad. In 10 cases there was evidence of a fracture in the radiograph taken seven to 14 days later. In these 10 cases all the fractures were of the intra-articular aspect of the radius (seven the radial neck and three the radial head). Nine of the 10 fractures were in patients with elevation of the anterior and posterior fat pads in the initial radiograph; in the other case only the anterior fat pad was elevated in the presenting radiograph. Two fractures were in patients aged under 16.

The fracture was visible in the radiographs at follow up because of bone resorption at the site of the fracture (nine cases) or a periosteal reaction at the radial neck (one case).

\footnotetext{
University Department of Radiodiagnosis, Royal Liverpool Hospital, Liverpool L69 3BX

D J W MOREWOOD, MRCP, FRCR, senior registrar
}

\begin{abstract}
All patients with traumatic effusion of the elbow in whom radiography at presentation does not show a fracture should undergo repeat radiography seven to 14 days later
\end{abstract}

\section{Discussion}

Under normal circumstances the posterior fat pad lies within the olecranon fossa and is not visible, whereas the anterior fat pad occupies the shallower anterior coronoid and radial fossae and may be visible ${ }^{2}$ (fig 1). Anatomically the fat is intracapsular but extrasynovial. ${ }^{3}$ With an intra-articular effusion the fat pads are displaced out of their fossae, so that the posterior fat pad becomes visible and the anterior fat pad elevated (fig 2).

At immediate supplementary radiographic examination Norell found 10 fractures not visible on routine examination of the elbow in 12 children with traumatic elbow effusion (the sites of the fractures were not mentioned). ${ }^{1}$ In another study of children with traumatic elbow effusion eight cases of small fracture were missed in the original radiographs. Of the remaining 12 children, who had follow up radiography at eight days to one month, five showed a periosteal reaction around the distal humerus, one due to osteitis and the four others to trauma. ${ }^{4}$

This study showed an incidence of fracture of $29 \%$ (10/35) in patients in whom an effusion but no fracture was visible on initial radiography. Though specific supplementary views taken at presentation-of the radial head or the radial head-capitellum, ${ }^{5}$ for example-might have shown several of these fractures at presentation, in practice the patient has usually left the radiography department before a radiologist could request these specific views.

Traumatic elbow effusion should be treated initially with a broad arm sling and analgesics. If a subsequent radiograph at seven to 14 

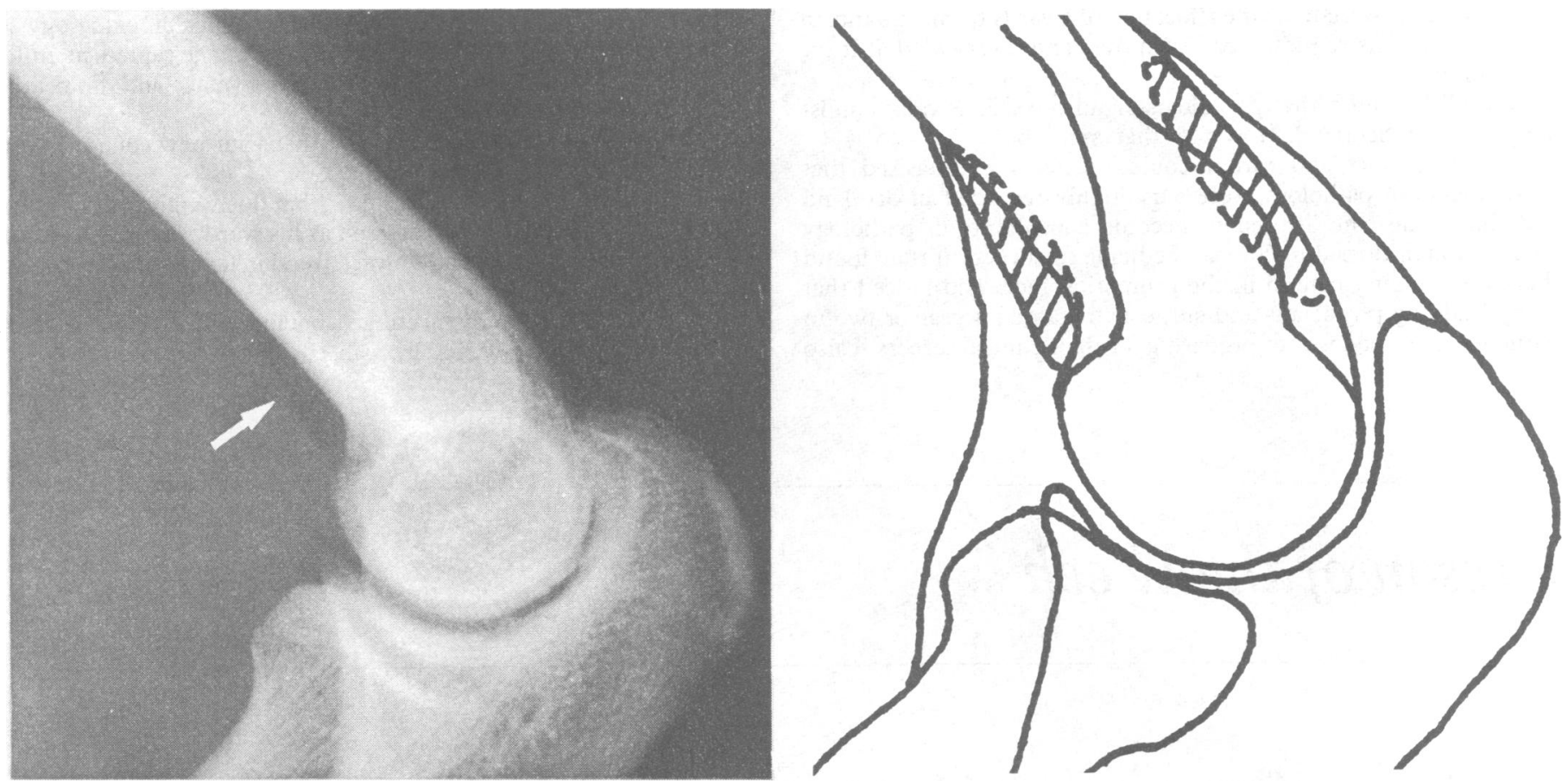

FIG 1-Left: Normal lateral radiograph of an elbow; only anterior fat pad is visible (arrow). Right: Line drawing of normal lateral radiograph of the elbow. Anterior fat pad (shaded) is partly visible and partly hidden in the radial and coronoid fossae. Posterior fat pad (shaded) is not visible as it lies within the olecronon fossa.

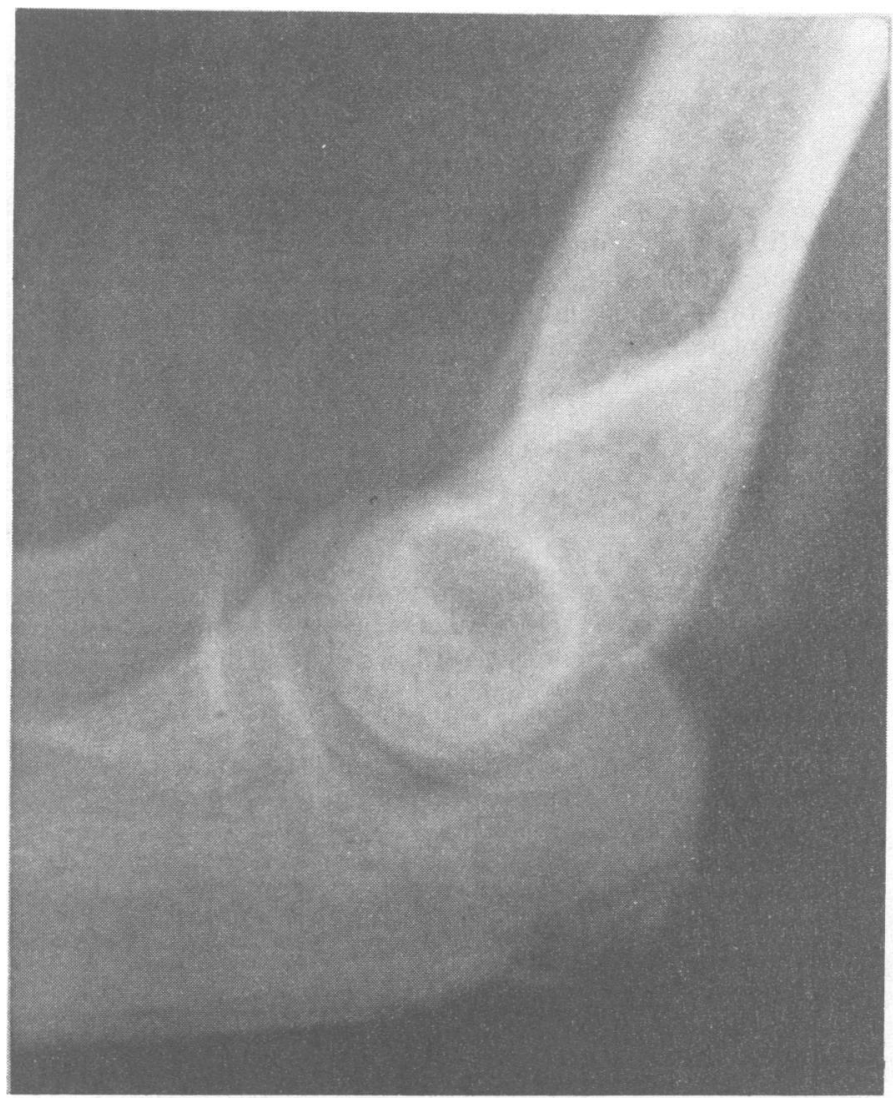

days shows no evidence of fracture then mobilisation can be started. If the repeat radiograph shows a fracture then a short period of further immobilisation may be necessary.

\section{References}

1 Norrell HG. Roentgenologic visualization of the extracapsular fat, its importance in the diagnosis of traumatic injuries to the elbow. Acta Radiologica 1954;42:205-10.

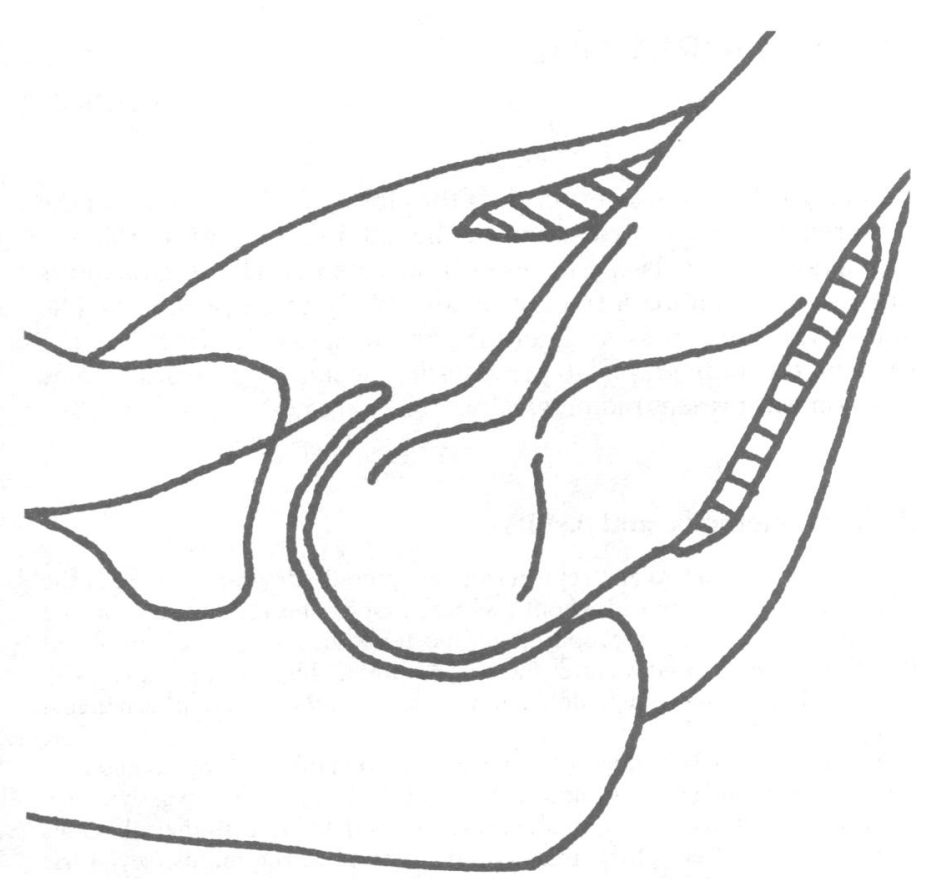

FIG 2-Left: Lateral radiograph of the elbow, showing elevation of anterior fat pad and visible posterior fat pad owing to an effusion. Above: Line drawing of lateral radiograph of the elbow, showing an effusion. Anterior fat pad (shaded) is elevated and posterior fat pad now visible.

2 Kohn AM. Soft tissue alterations in elbow trauma. American foumal of Roentgenology 1957;82:867-74.

3 Bledsoe RC, Izenstark JL. Displacement of fat pads in disease and injury of the elbow. Radiology 1959;73:717-24.

4 Bohrer SP. The fat pad sign following elbow trauma. Its usefulness and reliability in suspecting "invisible" fracture. Clin Radiol 1970;21:90-4.

5 Greenspan A, Norman A, Resen H. Radial head capitellum view in elbow trauma. Clinical application and radiographic-anatomical correlation. American foumal of Roentgenology 1984;143:355-9.

(Accepted 16 March 1987) 